\title{
Direct and Moderating Causal Effects of Network Support on Sleep Quality: Findings From the UC Berkeley Social Network Study
}

\author{
Stephanie Child, PhD, $\mathrm{MPH}^{1, \bullet} \cdot$ Emily H. Ruppel, $\mathrm{MA}^{2} \cdot$ Mia Zhong, $\mathrm{MA}^{3} \cdot$ Leora Lawton, $\mathrm{PhD}^{4}$
}

Published online: 24 September 2020

(C) Society of Behavioral Medicine 2020. All rights reserved. For permissions, please e-mail: journals.permissions@oup.com.

\begin{abstract}
Background Sleep is an important, restorative behavior for health, yet many adults report troubled sleep. The existence of a support network may be beneficial for sleep quality, including as a buffer for stressful events, yet few studies have examined these relationships longitudinally.

Purpose To examine the causal effect of changes in personal network support on sleep quality both directly and as a buffer of negative life events among young and older adults.

Methods The UC Berkeley Social Network survey collected data from young (21-30 year old, $n=475)$ and late middle-age (50-70 year old, $n=637$ ) adults across three waves between 2015 and 2018. Participants reported on personal network characteristics, negative life events, and number of nights with trouble falling and staying asleep. Fixed effects models are used to examine causal relationships among each age cohort.

Results Direct effects of network support on sleep quality were observed among older adults. Insufficient practical support predicted higher rates of trouble falling asleep (incident rate ratio $[\mathrm{IRR}]=1.40, p<.01$ ), while a desire for more social companions predicted lower rates of trouble staying asleep $(\mathrm{IRR}=0.81, p<.01)$. Buffering effects of network support on sleep quality were observed
\end{abstract}

Stephanie Child

schild07@gmail.com

1 Department of Social and Behavioral Science, Harvard TH Chan School of Public Health, 677 Huntington Avenue, Boston, MA 02115, USA

2 Department of Sociology, University of California, Berkeley, 410 Barrows Hall, Berkeley, CA 94720, USA

3 Department of Demography, University of California, Berkeley, 2232 Piedmont Avenue, Berkeley, CA 94720, USA

4 Berkeley Population Center, University of California, Berkeley, 360 Barrows Hall, Berkeley, CA 94720, USA among young adults. Changes in partnership status buffer the negative effects of the death of a close tie on trouble falling asleep (IRR $=0.75, p<.01$ ) and persistent difficulties paying bills on trouble staying asleep (IRR $=0.45, p<0.001)$ among young adults.

Conclusions This study provides evidence for the direct and buffering role of network support on sleep quality. Our results indicate that efforts to improve sleep quality should address personal networks and the support they provide, perhaps especially during times of stress for younger adults.

Keywords: Personal networks $\cdot$ Social support $\cdot$ Causal inference $\cdot$ Sleep quality

"Lay down last night Lord I could not get no rest. My mind was wandering like the wild geese in the west." -Traditional

\section{Introduction}

Sleep is an important aspect of overall well-being for physical and emotional health, as well as the ability to function effectively and enjoyably in the social, work, and family spheres of daily life. The personal networks inherent in those spheres have long been shown to be important for health and well-being via the support and resources that stem from those relationships [1]: recent research suggests that these networks may be important for sleep-related issues [2, 3]. Indeed, having social support is associated with a variety of sleep outcomes, including improved sleep quality, less disturbed sleep, and less insomnia [3-5].

As a restorative health behavior, sleep counters the physiological, behavioral, cognitive, and emotional experiences that accumulate during the day [6, 7]. Yet, getting a good night's sleep can be elusive: one third of 
adults report less than $7 \mathrm{hr}$ of sleep per night, despite recommendations for 7-9 hr per night [8]. Subsequently, deprivation of sleep quality and quantity has been associated with inflammatory disease risk, diabetes, cardiovascular disease, hypertension, and all-cause mortality [9-11]. Sleep quality is also hypothesized to be a factor related to the onset of cognitive decline and Alzheimer's disease $[12,13]$.

Given the detrimental effects of poor sleep on health, the sleep quality of Americans has drawn public health concern, especially given that $15 \%-20 \%$ of the adult U.S. population experiences chronic sleep issues [14, 15]. Prescribed and over-the-counter drugs are not recommended for long-term use and as such are a mismatch for chronic sleep disorders [16], so it is important to understand factors that would ameliorate its impact. While nondrug approaches are likely to lead to better resolutions over the long run, suggested approaches emphasize individual behavior rather than contextual factors [17]. However, much like similar health-related behaviors, identifying situational factors, such as social support, may lead to more appropriate responses to chronic sleep problems. In this paper, we explore how one's personal networks of family, friends, and coworkers directly or indirectly affect the ability of a person to fall and stay asleep.

\section{Networks, Social Support, and Sleep Quality}

An early study on the role of social support for life stress and health defined support broadly as information that leads someone to believe they are "cared for, loved, esteemed, and a member of a network of mutual obligations ([18], p. 300)." Findings from this seminal paper suggest that the support and resources available through social networks not only prevent troubled sleep but may also reduce the severity of troubled sleep. One possible direct link between networks and better sleep quality is that, from an evolutionary perspective, being surrounded by or in close proximity to others provides safety from potential predators or other dangers. Similarly, network support may provide reassurance about the ability to resolve current issues in one's life, particularly given that rumination occurring before sleep often leads to delays in sleep onset.

While a personal network consisting of friends is highly desirable, the presence of a romantic partner fills a unique position in the network. A romantic partner reflects a commitment, companionship, and shared goals and values, all of which act to reduce vulnerability, enhance security, and also to improve one's emotional, physical, and financial well-being [19]. All of these benefits of partnership are likely to be associated with better sleep quality [20]. A valuable feature of romantic relationships is stability, so changes in partnership status may have three pathways to affecting sleep. First, losing a partner can be associated with depression and stress [21], likely due to the loss of affection, companionship, a confidant, and instrumental support, all of which can affect sleep patterns. Second, gaining a partner may be associated with enhanced well-being and better sleep because of this. Third, there may also be negative effects resulting from the presence of network ties, for example, when relationships are particularly stressful, burdensome, or otherwise difficult. Even new and positive romantic partnerships may negatively affect sleep quality through sleep hygiene, such as irregular sleep schedules, arising from a partners' snoring, getting accustomed to a different room, or sharing a bed.

\section{Sleep During Difficult Times}

Negative life experiences and events can also contribute to poor sleep quality, a conceptual relationship originating with Adolph Meyer and further explored during the mid20th century [22]. Researchers examining psychological pathways linking life events and sleep quality have found that life changes cause sleep disorders and/or chronic insomnias via the aroused stress, rumination, and anxiety they cause $[23,24]$. In their pioneer retrospective study, Healey et al. [25] compared 31 chronic insomniacs with 31 good sleepers and found that the insomniacs experienced more stressful life events - particularly unpleasant and related to loss - during the year insomnia started. Similar patterns are seen in studies focusing on experiences after catastrophic disasters. Importantly, we note that the general indices of stress employed in these studies often include the loss of a network partner. Here, it is important to underscore mental health, including depression and anxiety, as an integral part of sleep quality and to reiterate that, in this study, we postulate that the pathway through which negative life events affect sleep is partly mediated through these mental states.

\section{Buffering Effects of Social Networks}

Thus far we have discussed how adequate social support can directly improve sleep quality, whereas negative life events can directly contribute to troubled sleep $[11,20,23$, 25-27]. It is also important to consider indirect pathways such that the impact of negative stressors on sleep quality can be mitigated by social support networks, which can reduce the overall effect of a stressor on well-being [28]. Because people rely on their social networks during major life transitions and events, these social ties are inherently crucial during difficult experiences as they enable balance and a sense of overall mental and physical well-being, leading to the ability to recover (i.e., resiliency). Cohen 
and Willis speculate that social support has direct benefits for well-being via embeddedness within a large social network, while buffering occurs when support is responsive to the needs elicited by the stressor [28], for example, by having confidants. However, another study among 18-60 year old adults found no moderation of perceived support on the relationship between general perceived stress and sleep quality [4].

\section{A Note on Measuring Social Experience}

Measuring social support and negative life events obligates unpacking because there are multiple ways of operationalizing these measures given that they are multifaceted concepts. One method for measuring social support is to create an index of support, for example, the Interpersonal Support Evaluation List or ISEL [29], a method often used in small sample sizes to gain statistical power. ISEL takes into account indicators that lead someone to believe that they are "cared for, loved, esteemed, and a member of a network of mutual obligations" [18]. Similarly, the Multidimensional Scale of Perceived Social Support [30] uses a Likert scale to incorporate the aspects of perceived support available across three specific domains: family, friends, and a significant other, asking respondents about their agreement to phrases such as "There is a special person who is around when I am in need" or "I can talk about my problems with my family." In this way, these scales describe what social support should look like and whether a person has met those external markers.

While there are many benefits to using these scales, an index can lose the granularity of lived experience of specific aspects of network sufficiency, that is, activity partners, confidants, and someone to provide help in a time of need. Having someone to do activities with indicates social connection or the lack of social isolation, thus filling the need for friendship, enjoyment, leisure, and relaxation such that engaging in enjoyable activities has been found to be associated with improved sleep quality [31]. Having a suitable number of confidants is a measure for having a way to work out problems and share concerns. Because a confidant relationship is founded on trust, having sufficient confidants signifies security, which is associated with a lower risk of depression [21, 32]. Finally, knowing that one has help if needed addresses the need to be interdependent rather than being overwhelmed with tasks or unmet needs and, therefore, has an emotional component, as well as its direct effect [30]. Despite having many network ties for these roles (an external marker), some individuals still feel like their needs are not met [33]. By asking about perceived network sufficiency, we can examine the discrepancy between availability and evaluation, leading to a marker of support as viewed by that individual.

As with measures of social support, measuring negative life events is not straightforward. Global measures of life stress, as used in Liu et al. [4] may be inappropriate given that support needs vary by stressor, which had led Cohen and Willis to suggest the examination of "specific stress-support linkages" (p. 348, emphasis added). Due to the difficulties of collecting data, assessments of negative life events for older adults are often either very general or consist of measures of single events, like unemployment. Other recent research on stressful life events and sleep focuses on catastrophic disasters [26, 34] rather than changes in status and personal experiences. However, because negative life events affect sleep through complex psychological tunnels, self-reported eventfulness can overlook less dramatic yet still important events that lead to mild but lasting burdens [24]. Moreover, because sleep is a personal, daily, repetitive, and restorative health behavior, research should benefit from elaborating the measurements of life events to a more individualized and diversified sphere. As such, in the current paper, we examine the buffering hypothesis by interacting three specific life events as sources of stress with three types of social support and with relationship status, allowing us a unique opportunity to examine how these events affect sleep quality.

\section{Hypotheses}

Previous research using short-term assessments of sleep has been limited in its ability to test for causal relationships between network support and sleep quality. Additionally, given that sleep quality has a reciprocal relationship between positive affect and the report of daily stressors [18, 31], it remains difficult to tease apart whether satisfaction with available social support leads to better sleep quality or if poor sleep quality leads to negative affect and subsequent dissatisfaction with ones' relationships. Furthermore, the majority of research on sleep quality has focused on clinical populations or older adults. Accordingly, this article contributes to the literature on network support, negative life events, and sleep quality in numerous ways. We use longitudinal panel rather than cross-sectional data to examine causal relationships. We examine multiple forms of negative stressors and three salient forms of network support, including partnership status for their effect on two different measures of sleep quality. Finally, because sleep quality is important throughout the life course, especially given that young adults are more susceptible to consequences related to sleep deficits than are older adults [35], we examine these relationships for two distinct age cohorts. 
To summarize, the current study tests three related hypotheses regarding the role of networks for sleep quality: (a) lack of network support predicts higher odds of experiencing troubled sleep, (b) lack of network support increases the severity of troubled sleep, and (c) the presence of network support buffers the effect of negative life events on likelihood and severity of troubled sleep. We extend this third hypothesis to examine whether changes in social support have a similar buffering effect for negative life events on sleep quality $[28,36]$.

\section{Methods}

\section{Sample}

Data come from the UC Berkeley Social Networks (UCNets) Study, a three-wave panel study conducted between 2015 and 2018 with rich data about changes in personal networks over time. The sample population is drawn from six counties in the San Francisco Bay Area and from two age cohorts at the time of enrollment: ages $21-30(n=475)$ and $50-70$ years old $(n=637)$. The older adult respondents were enrolled in the study via address-based sampling (ABS) and, as part of the original protocol to test mode effects [37], were randomly assigned to either a face-to-face (FTF) interview for survey completion or a nearly identical online survey. Sensitive questions in the UCNets data were collected using self-administered tools in the FTF model, so the responses avoid stigma or bias around asking and answering personal questions. However, the younger cohort proved difficult to reach and, in addition to the ABS method and random assignment to either an FTF interview or online survey completion, two thirds of the younger adult sample were recruited using a targeted Facebook advertisement strategy and, to a lesser extent, via respondent-driven referral $(n=37)$. Because of this modified and predominantly online sampling strategy, most of the younger cohort were directed to complete the online survey. In all analyses, we control for mode of survey (web vs. FTF) as a time-varying covariate.

\section{Measures}

\section{Dependent Variables}

Sleep quality consists of two measures that assess classic symptoms of insomnia: number of nights with difficulty falling asleep and number of nights with difficulty staying asleep. In the survey, participants were asked: "In an average week, about how many nights do you have trouble falling asleep?" and "About how many nights in the week do you find yourself waking up in the middle of the night and not easily able to fall back asleep?" Valid response options range from zero to seven nights. The distribution for each of these items was negatively skewed among both age cohorts, with a substantial proportion of individuals who answered zero nights.

\section{Independent Variables}

Network support was measured in two ways: satisfaction with support provided and romantic partnership status, thus incorporating both network sufficiency and intimate, familial support. Network sufficiency was measured with three questions about whether participants wished they had more people (a) to talk with about personal concerns (i.e., confidants), (b) to get together with socially (i.e., social companions), and (c) who could help with things like work around the home (i.e., practical help). Response options for each of the three questions were "I know enough already" (coded as "0" and referred to as "No") and "I wish I knew more" (coded as "1" and referred to as "Yes"). Relationship status was dichotomized into respondents who were currently in a relationship (i.e., partnered or married) and those who were not currently in a relationship.

\section{Negative life events}

Respondents were asked a variety of questions to assess whether they had experienced a significant life event or transition since the last time they were interviewed (or in the year prior to the first survey). These included persistent trouble with paying bills, death of a friend or family member, or a persisting major break with a nonromantic network tie, such as a relative or close friend. For each of these predictors, responses were coded as either "yes" $=1$ or "no" $=0$. For items about persistent troubles (e.g., bills and break in relationship), respondents were first asked whether they had experienced the event within the past year or so and, then, whether this was still currently a problem. Respondents who indicated "yes" to both questions were coded as " 1 " for these items and " 0 " if this event was no longer a problem. Finally, because we are interested in the ability of network support, including relationship status, to moderate the experience of a negative life event, we excluded cases where the death of a close tie happened to be a spouse or romantic partner given that their death would also result in a change of relationship status. This resulted in the exclusion of 11 older adults and 1 younger adult from the fixed effects analyses. 


\section{Analytic Approach}

This paper uses fixed effects modeling procedures to assess the causal effects of negative life events and social support on sleep quality, including as these factors interact with one another. Fixed effects models are widely considered the "gold standard" for causal inference from panel data [38, 39] as they control for all unmeasured time-invariant unit characteristics (e.g., respondent personality traits that could confound the associations under analysis) by modeling the effects of within-individual changes between waves in each independent variable on the dependent variable rather than conflating within- and between-individual patterns. As such, it is not necessary to adjust for social and demographic characteristics that are believed to be relatively stable (e.g., gender and race). While the statistical efficiency of these models is substantially reduced, they compensate for this inefficiency through their capacity to isolate causal relationships, reducing potential spuriousness by discarding between-unit variance. Weights based on the demographic composition of Wave 3 were applied to all models.

We first use descriptive statistics to examine the weighted sample characteristics by age cohort. We next use two types of fixed effects models to measure the causal effects of social support and negative life events on sleep quality, including negative events as moderated by social support. To examine our first research question, we use logistic fixed effects regression models to estimate the effects of social support and negative life events on the presence of any sleep problems, operationalized as the likelihood that respondents had trouble falling or staying asleep on one or more nights. Fixed effects logistic regression results are presented in Table 2 . We originally examined these models separately among both cohorts. However, because we observed a similar pattern of results among young and older adults, we collapsed these into a pooled sample and present one model for each outcome for all the respondents.

Our second research question focuses on the effects of social support and negative life events on the severity of sleep problems, operationalized as the count of nights per week that respondents struggled to fall or stay asleep. These results are presented by cohort in Tables 3 and 4 . Fixed effects Poisson models were used to account for the nonnormal distribution of our dependent variable and robust standard errors were applied to correct for overdispersion. Negative binomial models were considered but, ultimately, were not used due to Allison and Waterman's [40] critique on the inappropriate estimation of within-unit changes among conditional negative binomial fixed effects modeling procedures, as well as the difficulty of testing moderation effects within the hybrid fixed and random effects negative binomial models that Allison offers as a possible solution ([35], p. 49-69; [41]).

\section{Sensitivity Analyses}

One notable limitation of Poisson fixed effects models is that they discard respondents reporting all zero outcomes (i.e., anyone who reported no sleep problems at each wave), potentially skewing the sample that they draw upon and overstating correlations between sleep problems and other variables. Across both cohorts, 442 respondents reported zero nights with difficulty falling asleep and 384 respondents reported zero nights with difficulty staying asleep at each wave, meaning that they were excluded from Poisson models. The aforementioned logistic regression models for the presence or absence of sleep problems serve as one check against the bias this exclusion may introduce. As an additional robustness check, we tested fixed effects linear regression models for the impact of life events and social support on the severity of sleep problems, treating the number of nights with trouble sleeping as a continuous outcome. These models are not presented here due to the data's violation of the normality assumption for linear regression, but they provide a rough estimate of associations between sleep problems and other variables in the full sample. While linear regression models did not produce significantly different results from the Poisson models, they suggest that sampling bias may lead Poisson fixed effects models to slightly overestimate the significance of negative life events' effects on the severity of sleep problems, encouraging a conservative interpretation of our Poisson results. As such, we focus our interpretation of the results from the Poisson effects models on estimators with large effect sizes and/or $p$-values less than .01 and use the standard $p$-value of less than .05 for the logistic effects models.

Additionally, we used the mathematical procedures recommended in Vaisey and Miles [39] to test key assumptions of fixed effects modeling, namely the assumption of consistent time trajectories and the assumption of unidirectional causation with no selection into treatment. This latter set of tests explore whether reciprocal or reverse causation may be in play and are preferable to the inclusion of lagged variables because, as Vaisey and Miles describe, fixed effect models can produce severely biased coefficients when lags are misspecified.

\section{Results}

\section{Sample Characteristics}

Weighted sample characteristics reflect the Bay Area population and are presented in Table 1. Among young adults at Wave 1, 60\% reported at least one night per 
Table 1. Weighted sample characteristics

\begin{tabular}{|c|c|c|}
\hline & $\begin{array}{l}21-30 \\
\text { year olds } \\
(n=475 \text { at } \\
\text { Wave } 1)\end{array}$ & $\begin{array}{l}50-70 \\
\text { Year Olds } \\
(n=637 \text { at } \\
\text { Wave } 1)\end{array}$ \\
\hline & \multicolumn{2}{|l|}{ Percentage } \\
\hline \multicolumn{3}{|c|}{ Percentage reporting difficulty falling asleep at Wave 1} \\
\hline 0 nights & 40.4 & 57.4 \\
\hline 1 night & 16.6 & 12.9 \\
\hline 2 nights & 11.9 & 7.5 \\
\hline 3 nights & 7.8 & 5.1 \\
\hline 4 nights & 4.4 & 2.3 \\
\hline 5 nights & 8.4 & 2.1 \\
\hline 6 nights & 1.2 & 1.8 \\
\hline 7 nights & 9.3 & 10.9 \\
\hline \multicolumn{3}{|c|}{ Percentage reporting difficulty staying asleep at Wave 1} \\
\hline 0 nights & 51.5 & 45.0 \\
\hline 1 night & 20.3 & 16.7 \\
\hline 2 nights & 9.0 & 9.1 \\
\hline 3 nights & 4.4 & 7.4 \\
\hline 4 nights & 4.1 & 2.6 \\
\hline 5 nights & 4.5 & 3.2 \\
\hline 6 nights & 0.2 & 1.8 \\
\hline 7 nights & 6.0 & 14.2 \\
\hline \multicolumn{3}{|c|}{ Indicators of social support at Wave 1} \\
\hline \multicolumn{3}{|l|}{$\begin{array}{l}\text { Respondents who wish for } \\
\text { more... }\end{array}$} \\
\hline Confidants & 31.1 & 26.3 \\
\hline Social companions & 65.5 & 46.9 \\
\hline Practical helpers & 31.3 & 26.9 \\
\hline Partnered or married & 64.2 & 70.0 \\
\hline \multicolumn{3}{|c|}{$\begin{array}{l}\text { Percentage ever reporting negative life events } \\
\text { (waves pooled) }\end{array}$} \\
\hline Death of close tie & 57.2 & 72.7 \\
\hline Persisting break with close tie & 56.3 & 41.5 \\
\hline Persisting trouble paying bills & 38.1 & 18.3 \\
\hline \multicolumn{3}{|l|}{ Demographics at Wave 1} \\
\hline Female & 50.8 & 51.6 \\
\hline \multicolumn{3}{|l|}{ Race/ethnicity $^{\mathrm{a}}$} \\
\hline White & $52.9 \%$ & $62.9 \%$ \\
\hline Black & $8.80 \%$ & $8.63 \%$ \\
\hline Latino & $24.7 \%$ & $12.4 \%$ \\
\hline Asian & $24.0 \%$ & $19.0 \%$ \\
\hline Other & $23.2 \%$ & $11.1 \%$ \\
\hline \multicolumn{3}{|l|}{ Educational attainment } \\
\hline Less than bachelor's & $61.1 \%$ & $56.1 \%$ \\
\hline Bachelor's degree & $28.0 \%$ & $24.6 \%$ \\
\hline More than bachelor's & $10.9 \%$ & $19.3 \%$ \\
\hline
\end{tabular}

${ }^{a}$ Not mutually exclusive. week with trouble falling asleep. Just over half reported no trouble staying asleep, while nearly a third reported trouble staying asleep on either one $(20.3 \%)$ or two $(9.0 \%)$ nights at Wave 1. Among the older cohort, more than half reported no trouble falling asleep (57\%). However, one quarter of the older cohort reported trouble staying asleep on at least one (17\%) or two (9\%) nights, and a substantial 14\% reported trouble staying asleep every night. These patterns were fairly consistent across all three time points. Regarding social network support, about a third of young adults reported a desire for more people to talk to about important matters $(31 \%)$ or ties who could provide practical help (31\%), while two thirds wished they had more people to get together with socially (social companions; 66\%). For older adults, one quarter wished for more people to talk to about important matters $(26 \%)$ or ties who could provide practical help (27\%), and about half wished they had more people to get together with socially $(47 \%)$. Two thirds of younger adults $(64 \%)$ were in a romantic partnership at Wave 1 compared to $70 \%$ of the older adults. More than half of younger adults experienced the death of a close tie $(57 \%)$ or a persisting break in a relationship $(56 \%)$, while more than a third experienced persistent trouble paying bills across the three waves $(38 \%)$, whereas more of the older cohort reported the death of a close tie (two thirds), but fewer, a fifth, reported persistent trouble with paying bills. Reflecting the San Francisco Bay Area demographic profile, a majority of the participants in the younger cohort were white $(53 \%)$ versus $63 \%$ of the older adults. Among the younger cohort, $61 \%$ had not completed a bachelor's degree compared to $56 \%$ of older adults).

\section{Direct Predictors of Any Sleep Problems}

To test our first hypothesis, fixed effects logistic regression models were used to examine the effect of changes in network support on the odds of troubled sleep on one or more nights among the combined sample of UCNets participants (Table 2). Changes in network support variables are associated with odds of difficulty staying asleep but not falling asleep. Changes in network sufficiency, that is, reporting a desire for more people to provide practical help, nearly doubles the odds of difficulty staying asleep (odds ratio $[\mathrm{OR}]=1.73,95 \%$ confidence interval [CI]: 1.10, 2.73; Table 2; Model 3). Additionally, change in partnership status (i.e., to being partnered or married) also doubles the odds of difficulty staying asleep $(\mathrm{OR}=2.26,95 \% \mathrm{CI}: 1.07,4.78$; Table 2; Model 3). 
Table 2. Fixed effects logistic regression models estimating the effect of social support on the presence of any sleep problems in the full UC Berkeley Social Networks Study sample ( $n=$ observations across three waves)

\begin{tabular}{|c|c|c|c|c|c|c|}
\hline & \multicolumn{2}{|c|}{$\begin{array}{l}\text { Difficulty falling asleep } \\
\text { OR }(95 \% \mathrm{CI})\end{array}$} & \multicolumn{4}{|c|}{ Difficulty staying asleep OR (95\% CI) } \\
\hline & $\begin{array}{l}\text { Model } 1 \\
(n=990)\end{array}$ & $\begin{array}{l}\text { Model } 2 \\
(n=983)\end{array}$ & $\begin{array}{l}\text { Model } 3 \\
(n=1,066)\end{array}$ & $\begin{array}{l}\text { Model } 4 \\
(n=1,060)\end{array}$ & $\begin{array}{l}\text { Model } 5 \\
(n=1,112)\end{array}$ & $\begin{array}{l}\text { Model } 6 \\
(n=1,143)\end{array}$ \\
\hline \multicolumn{7}{|l|}{ Social support } \\
\hline \multicolumn{7}{|l|}{ Desire for more... } \\
\hline Confidants & $\begin{array}{l}0.94 \\
(0.61,1.45)\end{array}$ & $\begin{array}{l}0.93 \\
(0.60,1.44)\end{array}$ & $\begin{array}{l}1.23 \\
(0.77,1.95)\end{array}$ & $\begin{array}{l}1.23 \\
(0.77,1.96)\end{array}$ & - & - \\
\hline Social companions & $\begin{array}{l}1.05 \\
(0.69,1.62)\end{array}$ & $\begin{array}{l}1.07 \\
(0.69,1.65)\end{array}$ & $\begin{array}{l}1.06 \\
(0.69,1.65)\end{array}$ & $\begin{array}{l}1.07 \\
(0.69,1.66)\end{array}$ & - & - \\
\hline Practical helpers & $\begin{array}{l}1.25 \\
(0.81,1.91)\end{array}$ & $\begin{array}{l}1.27 \\
(0.83,1.96)\end{array}$ & $\begin{array}{l}1.73^{*} \\
(1.10,2.73)\end{array}$ & $\begin{array}{l}1.72^{*} \\
(1.09,2.73)\end{array}$ & $\begin{array}{l}2.24 * * \\
(1.38,3.64)\end{array}$ & - \\
\hline Partnered or married & $\begin{array}{l}0.99 \\
(0.53,1.83)\end{array}$ & $\begin{array}{l}1.01 \\
(0.54,1.88)\end{array}$ & $\begin{array}{l}2.26 * \\
(1.07,4.78)\end{array}$ & $\begin{array}{l}2.28 * \\
(1.07,4.83)\end{array}$ & - & $\begin{array}{l}3.46 * * \\
(1.63 \\
7.38)\end{array}$ \\
\hline \multicolumn{7}{|l|}{ Negative life events } \\
\hline Death of a close tie & & $\begin{array}{l}1.27 \\
(0.91,1.78)\end{array}$ & & $\begin{array}{l}1.06 \\
(0.77,1.46)\end{array}$ & - & - \\
\hline Persisting break with a close tie & & $\begin{array}{l}1.10 \\
(0.74,1.65)\end{array}$ & & $\begin{array}{l}0.98 \\
(0.66,1.45)\end{array}$ & $\begin{array}{l}1.38 \\
(0.88,2.13)\end{array}$ & - \\
\hline Persisting difficulty paying bills & & $\begin{array}{l}0.97 \\
(0.52,1.81)\end{array}$ & & $\begin{array}{l}1.33 \\
(0.68,2.62)\end{array}$ & - & $\begin{array}{l}2.61 * \\
(1.01 \\
6.72)\end{array}$ \\
\hline \multicolumn{7}{|l|}{ Interactions } \\
\hline $\begin{array}{l}\text { Persisting break with a close tie } \times \\
\text { Desire for more practical help }\end{array}$ & & & & & $\begin{array}{l}0.39^{*} \\
(0.18,0.86)\end{array}$ & \\
\hline $\begin{array}{l}\text { Persisting difficulty paying bills } \times \\
\text { Partnered status }\end{array}$ & & & & & & $\begin{array}{l}0.28 * \\
(0.09 \\
0.86)\end{array}$ \\
\hline
\end{tabular}

$C I$ confidence interval; $O R$ odds ratio.

${ }^{*} p<.05,{ }^{* *} p<.01, * * * p<.001$.

\section{Direct Predictors of Severity in Trouble Falling Asleep}

To test part of our second hypothesis, fixed effects Poisson regression models were used to examine the effect of network support on the severity of trouble falling asleep among participants who reported any difficulties falling asleep across the three waves, stratified by age cohort (Table 3). Among young adults who reported sleep troubles, no changes in network support or negative life events predicted the severity of trouble falling asleep. Among older adults and adjusting for negative life events, a desire for more practical help predicted a higher rate of nights with trouble falling asleep (IRR $=1.40,95 \%$ CI: 1.15, 1.69; Table 3; Model 6). Additionally, a persisting break with a close tie predicted greater severity of trouble falling asleep among older adults (IRR $=1.33,95 \%$ CI: 1.08, 1.64: Table 3; Model 6).

\section{Direct Predictors of Severity in Trouble Staying Asleep}

As part of our second hypothesis, fixed effects Poisson regression models were also used to examine the effect of network support on the severity of trouble staying asleep among participants who reported any trouble staying asleep across the three waves, stratified by age cohort (Table 4). Among the younger cohort who experienced any trouble falling asleep, persistent difficulty paying bills was predictive of a higher rate of nights with difficulty staying asleep $(\mathrm{IRR}=1.45,95 \% \mathrm{CI}: 1.21,1.74$; Table 4; Model 2). Among the older cohort and adjusting for negative life events, changes in network sufficiency to desiring more social companions predicted a lower rate of severity of trouble staying asleep (IRR $=0.81,95 \% \mathrm{CI}$ : 0.69, 0.95; Table 4; Model 6). Finally, among the older cohort, a persisting break with a close tie was predictive of greater severity of nights with difficulty staying asleep $(\mathrm{IRR}=1.33,95 \%$ CI: 1.13, 1.57; Table 4; Model 6).

\section{Buffering Effects}

Our third hypothesis underscores the ability of network support to buffer the negative effect of negative life events on troubled sleep. To test this, we examine 
Table 3. Fixed effects Poisson regression models estimating the effect of social support on the severity of difficulty falling asleep by age cohort ( $n=$ observations across three waves)

\begin{tabular}{|c|c|c|c|c|c|c|c|c|}
\hline & \multicolumn{4}{|c|}{$21-30$ year olds IRR $(95 \% \mathrm{CI})$} & \multicolumn{4}{|c|}{$50-70$ year olds IRR $(95 \% \mathrm{CI})$} \\
\hline & $\begin{array}{l}\text { Model } 1 \\
(n=883)\end{array}$ & $\begin{array}{l}\text { Model } 2 \\
(n=879)\end{array}$ & $\begin{array}{l}\text { Model } 3 \\
(n=898)\end{array}$ & $\begin{array}{l}\text { Model } 4 \\
(n=888)\end{array}$ & $\begin{array}{l}\text { Model } 5 \\
(n=913)\end{array}$ & $\begin{array}{l}\text { Model } 6 \\
(n=909)\end{array}$ & $\begin{array}{l}\text { Model } 7 \\
(n=965)\end{array}$ & $\begin{array}{l}\text { Model } 8 \\
(n=952)\end{array}$ \\
\hline \multicolumn{9}{|l|}{ Social support } \\
\hline \multicolumn{9}{|l|}{ Desire for more... } \\
\hline Confidants & $\begin{array}{l}0.84^{*} \\
(0.72,0.97)\end{array}$ & $\begin{array}{l}0.86 \\
(0.73,1.00)\end{array}$ & - & $\begin{array}{l}0.81 * * \\
(0.71,0.93)\end{array}$ & $\begin{array}{l}1.19 \\
(0.97,1.46)\end{array}$ & $\begin{array}{l}1.05 \\
(0.81,1.37)\end{array}$ & - & $\begin{array}{l}1.16 \\
(0.86 \\
1.55)\end{array}$ \\
\hline Social companions & $\begin{array}{l}1.06 \\
(0.92,1.22)\end{array}$ & $\begin{array}{l}1.04 \\
(0.89,1.22)\end{array}$ & - & - & $\begin{array}{l}0.95 \\
(0.78,1.16)\end{array}$ & $\begin{array}{l}0.95 \\
(0.77,1.15)\end{array}$ & - & - \\
\hline Practical helpers & $\begin{array}{l}0.91 \\
(0.78,1.06)\end{array}$ & $\begin{array}{l}0.90 \\
(0.76,1.07)\end{array}$ & - & - & $\begin{array}{l}1.36^{*} \\
(1.12,1.64)\end{array}$ & $\begin{array}{l}1.40 * * \\
(1.15,1.69)\end{array}$ & - & - \\
\hline Partnered or married & $\begin{array}{l}0.78^{*} \\
(0.62,0.99)\end{array}$ & $\begin{array}{l}0.78 \\
(0.61,1.00)\end{array}$ & $\begin{array}{l}1.03 \\
(0.82,1.28)\end{array}$ & - & $\begin{array}{l}1.35 \\
(0.80,2.26)\end{array}$ & $\begin{array}{l}1.38 \\
(0.78,2.45)\end{array}$ & $\begin{array}{l}1.38 \\
(0.81,2.35)\end{array}$ & - \\
\hline \multicolumn{9}{|l|}{ Negative life events } \\
\hline Death of a close tie & & $1.01(0.90,1.14)$ & $\begin{array}{l}1.24 * * \\
(1.07,1.44)\end{array}$ & - & & $\begin{array}{l}1.00 \\
(0.88,1.16)\end{array}$ & $\begin{array}{l}0.99 \\
(0.76,1.27)\end{array}$ & - \\
\hline $\begin{array}{l}\text { Persisting break with a } \\
\text { close tie }\end{array}$ & & $\begin{array}{l}0.92 \\
(0.79,1.08)\end{array}$ & - & $\begin{array}{l}0.79 * \\
(0.65,0.96)\end{array}$ & & $\begin{array}{l}1.33 * * \\
(1.08,1.64)\end{array}$ & - & $\begin{array}{l}1.27^{*} \\
(1.02 \\
1.59)\end{array}$ \\
\hline $\begin{array}{l}\text { Persisting difficulty } \\
\text { paying bills }\end{array}$ & & $\begin{array}{l}1.05 \\
(0.88,1.26)\end{array}$ & - & - & & $\begin{array}{l}0.98 \\
(0.76,1.26)\end{array}$ & - & - \\
\hline \multicolumn{9}{|l|}{ Interactions } \\
\hline $\begin{array}{l}\text { Death of a close tie } \times \\
\text { Partnered status }\end{array}$ & & & $\begin{array}{l}0.75^{* *} \\
(0.61,0.91)\end{array}$ & & & & $\begin{array}{l}0.98 \\
(0.73,1.31)\end{array}$ & \\
\hline $\begin{array}{l}\text { Persisting break with a } \\
\text { close tie } \times \text { Desire for } \\
\text { more confidants }\end{array}$ & & & & $\begin{array}{l}1.36^{*} \\
(1.02,1.81)\end{array}$ & & & & $\begin{array}{l}0.87 \\
(0.59 \\
1.27)\end{array}$ \\
\hline
\end{tabular}

$C I$ confidence interval.

$* p<.05, * * p<.01, * * * p<.001$.

interaction effects in both the logistic and Poisson fixed effects models. Among the fixed effects logistic models, which examine for the presence of any troubled sleep among the pooled sample, the desire for more practical help moderates the association between experiencing a chronic break and trouble staying asleep. Among participants who have not experienced a chronic break, those with no desire for more practical support experience better sleep than those who do wish for more practical support. However, this gap diminishes among those who have experienced a chronic break $(\mathrm{OR}=0.39,95 \% \mathrm{CI}$ : 0.18, 0.86; Table 2; Model 5; pattern similar to Fig. 1). Similarly, the gap between being in a romantic relationship versus not narrows in the presence of persisting trouble paying bills such that there are no significant differences in difficulty staying asleep by partnered status among those with trouble paying bills (OR $=0.28,95 \%$ CI: 0.09, 0.86; Table 2; Model 6; Fig. 1).

We examine the severity of troubled sleep among those who report any trouble sleeping using fixed effects
Poisson models, finding that the ability of network support characteristics to moderate the severity of troubled sleep was observed only among the younger cohort. For young adults, positive changes in partnership status appear to buffer the negative effects of the death of a close tie on the severity of trouble falling asleep (IRR $=0.75$, 95\% CI: 0.61, 0.91; Table 3; Model 3; Fig. 2). Moreover, young adults who become partnered have greater severity of trouble staying asleep when no persisting difficulties with paying bills are reported yet have lower severity with trouble staying asleep in the presence of persistent difficulties paying bills (IRR $=0.45,95 \%$ CI: $0.33,0.63$; Table 4; Model 3; Fig. 3).

\section{Discussion}

Social relationships and the support they provide during times of duress are hypothesized to be important for sleep quality, so this study, and its ability to test causal 
Table 4. Fixed effects Poisson regression models estimating the effect of social support on the severity of difficulty staying asleep by age cohort ( $n=$ observations across three waves)

\begin{tabular}{|c|c|c|c|c|c|c|c|c|}
\hline & \multicolumn{4}{|c|}{$21-30$ year olds IRR $(95 \% \mathrm{CI})$} & \multicolumn{4}{|c|}{$50-70$ year olds IRR $(95 \% \mathrm{CI})$} \\
\hline & $\begin{array}{l}\text { Model } 1 \\
(n=739)\end{array}$ & $\begin{array}{l}\text { Model } 2 \\
(n=735)\end{array}$ & $\begin{array}{l}\text { Model } 3 \\
(n=755)\end{array}$ & $\begin{array}{l}\text { Model } 4 \\
(n=746)\end{array}$ & $\begin{array}{l}\text { Model } 5 \\
(n=1,183)\end{array}$ & $\begin{array}{l}\text { Model } 6 \\
(n=1,177)\end{array}$ & $\begin{array}{l}\text { Model } 7 \\
(n=1,258)\end{array}$ & $\begin{array}{l}\text { Model } 8 \\
(n=1,226)\end{array}$ \\
\hline \multicolumn{9}{|l|}{ Social support } \\
\hline \multicolumn{9}{|l|}{ Desire for more... } \\
\hline Confidants & $\begin{array}{l}0.98 \\
(0.80,1.20)\end{array}$ & $\begin{array}{l}0.93 \\
(0.76,1.14)\end{array}$ & - & - & $\begin{array}{l}1.08 \\
(0.94,1.25)\end{array}$ & $\begin{array}{l}0.98 \\
(0.83,1.16)\end{array}$ & - & - \\
\hline Social companions & $\begin{array}{l}0.95 \\
(0.75,1.19)\end{array}$ & $\begin{array}{l}0.89 \\
(0.69,1.14)\end{array}$ & - & - & $\begin{array}{l}0.84^{*} \\
(0.72,0.99)\end{array}$ & $\begin{array}{l}0.81^{* *} \\
(0.69,0.95)\end{array}$ & - & - \\
\hline Practical helpers & $\begin{array}{l}1.12 \\
(0.95,1.33)\end{array}$ & $\begin{array}{l}1.25^{*} \\
(1.03,1.52)\end{array}$ & - & $\begin{array}{l}1.42 * * \\
(1.10,1.82)\end{array}$ & $\begin{array}{l}1.11 \\
(0.96,1.29)\end{array}$ & $\begin{array}{l}1.15 \\
(0.99,1.34)\end{array}$ & - & $\begin{array}{l}1.18^{*} \\
(1.00 \\
1.38)\end{array}$ \\
\hline Partnered or married & $\begin{array}{l}0.88 \\
(0.65,1.18)\end{array}$ & $\begin{array}{l}0.85 \\
(0.63,1.16)\end{array}$ & $\begin{array}{l}1.51^{*} \\
(1.01,2.27)\end{array}$ & - & $\begin{array}{l}1.73^{*} \\
(1.00,2.99)\end{array}$ & $\begin{array}{l}1.80 \\
(0.99,3.25)\end{array}$ & $\begin{array}{l}1.81^{*} \\
(1.02,3.19)\end{array}$ & - \\
\hline \multicolumn{9}{|l|}{ Negative life events } \\
\hline Death of a close tie & & $\begin{array}{l}1.10 \\
(0.90,1.33)\end{array}$ & - & - & & $\begin{array}{l}0.99 \\
(0.87,1.12)\end{array}$ & - & - \\
\hline Persisting break with a close tie & & $\begin{array}{l}1.14 \\
(0.93,1.39)\end{array}$ & - & $\begin{array}{l}1.37 * * \\
(1.09,1.73)\end{array}$ & & $\begin{array}{l}1.33 * * \\
(1.13,1.57)\end{array}$ & - & $\begin{array}{l}1.39 * * * \\
(1.17 \\
1.65)\end{array}$ \\
\hline Persisting difficulty paying bills & & $\begin{array}{l}1.45 * * * \\
(1.21,1.74)\end{array}$ & $\begin{array}{l}2.20 * * * \\
(1.76,2.74)\end{array}$ & - & & $\begin{array}{l}0.97 \\
(0.79,1.18)\end{array}$ & $\begin{array}{l}0.96 \\
(0.75,1.24)\end{array}$ & - \\
\hline \multicolumn{9}{|l|}{ Interactions } \\
\hline $\begin{array}{l}\text { Persisting difficulty paying bills } \\
\times \text { Partnered status }\end{array}$ & & & $\begin{array}{l}0.45^{* * *} \\
(0.33,0.63)\end{array}$ & & & & $\begin{array}{l}1.04 \\
(0.68,1.59)\end{array}$ & \\
\hline $\begin{array}{l}\text { Persisting break with a close tie } \times \\
\text { Desire for more practical help }\end{array}$ & & & & $\begin{array}{l}0.60^{*} \\
(0.40,0.91)\end{array}$ & & & & $\begin{array}{l}0.75^{*} \\
(0.69 \\
1.64)\end{array}$ \\
\hline
\end{tabular}

CI confidence interval.

$* p<.05, * * p<.01, * * * p<.001$.

direct and indirect relationships using rich, longitudinal data on networks, life stressors, and sleep, contributes to the body of literature regarding troubled sleep. Overall, the findings provide support for both direct and moderating effects of network support on future sleep quality among young and older adults. Consistent with our first and second hypotheses for direct effects and previous literature $[2,3]$, network insufficiency, particularly the desire for more practical support, was predictive of both higher odds of troubled sleep and greater severity of troubled sleep. Changes in network appraisal of available support may reflect two distinct events: actual network loss and recent experiences in which support that was previously perceived as adequate is no longer viewed as such. Either scenario may provoke negative emotions or psychological states, including depression, anxiety, and stress, all of which have subsequent effects on sleep. Indeed, perceived support has been shown to mediate the path between rumination and subsequent depression [42]. Furthermore, decreases in perceived support over time are associated with increased depression [43]. The current study extends upon these findings to indicate that changes in perceived network support sufficiency may have downstream consequences for sleep quality as well.

However, additional findings were in the opposite direction from the hypothesis. Among older adults, the desire for more people to socialize with was predictive of fewer nights with difficulties staying asleep (the most problematic sleep issue among this cohort in the current sample). Based on prior research, loneliness and poor perceived support should be associated with poor sleep quality [36]. Similarly, we hypothesized that network insufficiency would be associated with more distress and, therefore, problematic sleep. The findings here suggest a different mechanism: the desire for more social companions, particularly among older adults, may also signal individuals who are healthy enough for active lives and are looking for more companions to spend time with doing various activities. Compared 


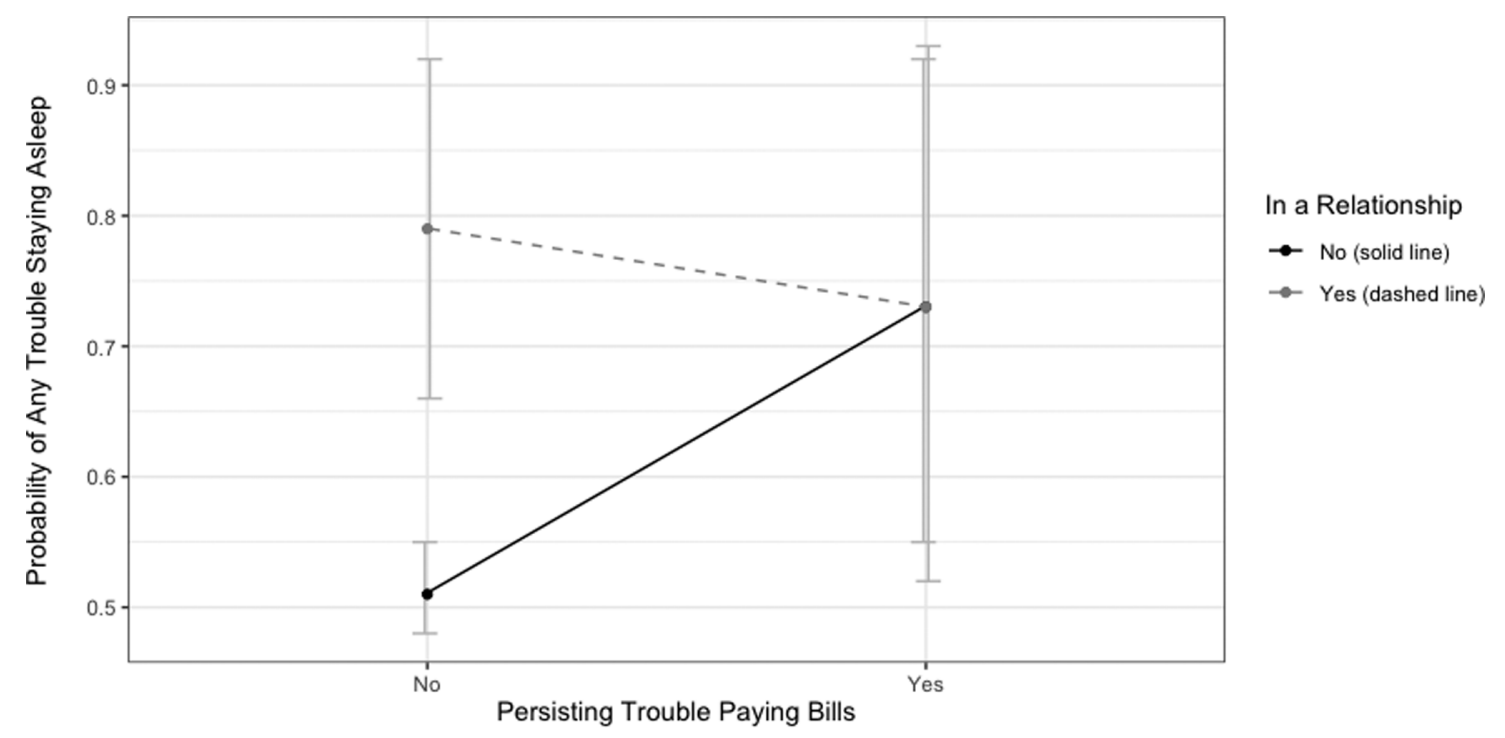

Fig. 1. Effect of persistent difficulties paying bills on troubled sleep by relationship status among the pooled sample.

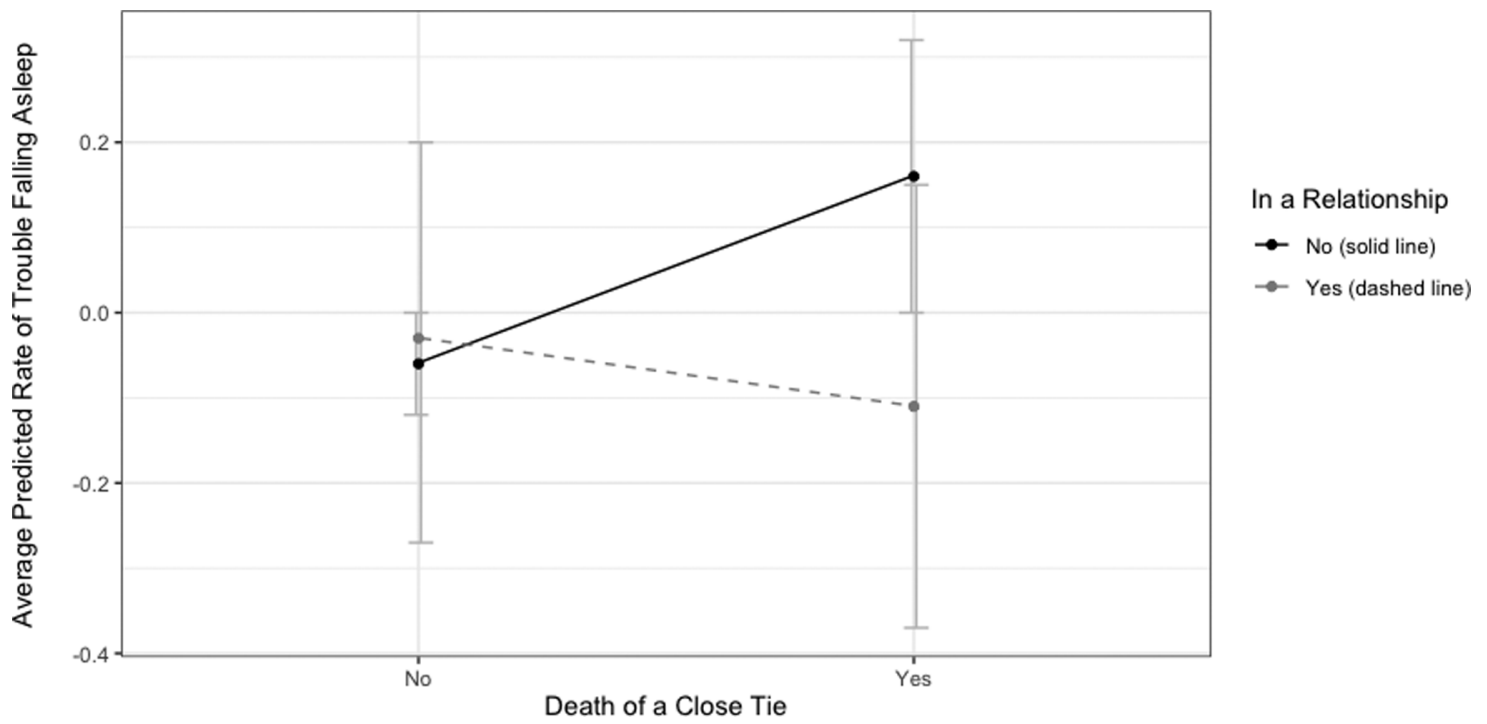

Fig. 2. Effect of death of a close tie on severity of trouble falling asleep by relationship status among young adults.

with young adults, older adults may be more likely to have friends or other network ties who are going out less frequently or who may not be geographically proximal. Previous research confirms that older adults who are more socially active report better quality sleep [44]. As such, the desire for more social companions may signal an increase in or the desire and ability for more social outings.

A change in partnership status, to being partnered or married, predicted poorer sleep quality, and trouble staying asleep in particular. While previous studies have indicated sleep quality is better among partnered individuals than nonpartnered individuals [20, 45], these results suggest that it is important to consider both positive and negative aspects of romantic partnerships that are associated with sleep characteristics [46, 47]. Furthermore, because the current findings indicate that the transition to being partnered is predictive of both higher odds and greater severity of difficulties sleeping, even positive aspects of one's network may have negative consequences for sleep quality. Recent changes in partnership status include additional situational changes, including changes in living arrangements and changes in sleeping routines, pointing to sleep quality that is now in part dependent upon another person [48], all of which may disrupt sleep. 


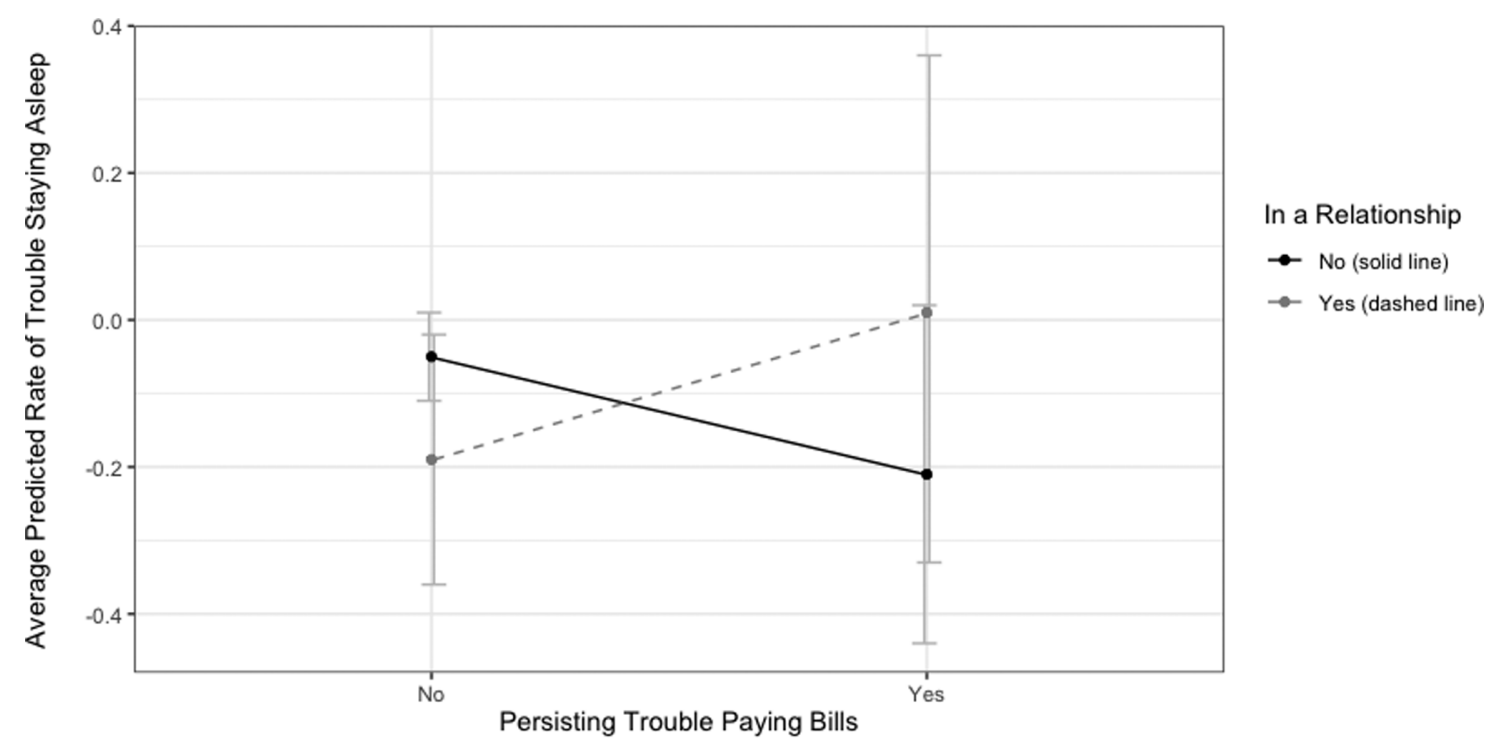

Fig. 3. Effect of persistent difficulties paying bills on severity of trouble staying asleep by relationship status among young adults.

In addition to direct effects of network support on troubled sleep, support is primarily hypothesized to promote sleep outcomes through its ability to mitigate the negative effects of stress on sleep quality. To test this relationship using a causal framework, we examined whether changes in support characteristics moderated the effect of three types of negative life events on sleep quality across three waves of the UCNets survey data. We find support for our third hypothesis: Partnership status and the desire for more practical help or confidants moderated relationships between stressful life events and troubled sleep, indicating that changes in network support affect sleep quality in conjunction with stressful events.

Becoming partnered or married moderated the relationship between sleep quality and two of the three negative events we tested as shown in each of the figures. On the whole, change to being in a partnered or married relationship predicted worse, if not similar, levels of troubled sleep in the absence of a negative event compared with those who were not in a relationship. This may be due to changes in sleep routines, cohabiting, and other challenges related to sharing sleep with another individual. However, being partnered or married also prevented worse sleep quality in the presence of a negative event. Indeed, in all three instances, the effect of a negative event worsens sleep quality among those who are not in a relationship (positive slope), whereas the effect of a negative event appears to be neutral for sleep quality among those who are recently partnered or married. This effect was seen most clearly in terms of the severity of troubled sleep (Figs. 2 and 3) rather than odds of any troubled sleep (Fig. 1). Thus, while changes to relationship status increase the odds of troubled sleep, these changes are protective against deleterious effects of stress on the severity of troubled sleep. While relationship status appeared to moderate the experience of stress broadly, across two types of negative experiences, the classic literature on buffering calls for greater specificity in terms of stressor and support linkages [28]. That is, identifying specific forms of support salient for certain types of stressors may be most fruitful.

Unlike among young adults, neither social support from the network nor partnership status appeared to moderate the effect of negative life events on severity of sleep quality among older adults. Older adults reported less trouble falling asleep than they did staying asleep, which may signal distinct reasons for each. For example, while trouble falling asleep may be due to rumination, anxiety, and poor sleep hygiene, trouble staying asleep or falling back to sleep after awaking may be due to additional physiological factors, such as waking in the middle of the night to use the restroom, noise, or discomfort and pain. Sleep quality associated with physiological factors may be less responsive to social support. As such, the factors associated with poor sleep quality among older adults may be less modifiable via network support.

\section{Limitations}

The findings should be interpreted in light of certain limitations. First, all data are self-reported and subject to recall and desirability bias. The measure for relationship or partner status is binary and, therefore, does not allow for nuances that may be related to sleep, such as 
relationship quality, or the desire for a romantic partnership. Furthermore, the UCNets survey did not use a previously validated measure of sleep quality. Instead, the outcomes captured are fairly crude changes in sleep quality (i.e., self-reported number of nights with sleep trouble), and future studies would be substantially improved with the use of validated and more sensitive measures of sleep quality, such as sleep actigraph data. As such, the findings and interpretations are conservative and likely underestimate the role of networks and negative life events on sleep quality. Second, the ability to compare findings from this study with prior research is limited due to differences in sleep quality measurement. Similarly, the findings are limited in generalizability to comparable demographic groups of adult urban residents. Third, differences in the sampling approach for each cohort (e.g., probability sampling for the older cohort and probability plus convenience sampling for the younger cohort) may have contributed to within-individual, nonrandom differences across the two samples. A previous paper describes these few differences among the younger cohort who were recruited via Facebook versus those recruited via probability sampling [49]. However, it is possible that other unmeasured, nonrandom differences within the sample exist.

As noted previously, it is necessary to interpret the current Poisson estimates conservatively given the sampling bias inherent to Poisson fixed effects modeling procedures. Poisson models exclude respondents reporting no change on the dependent variable, which includes respondents who reported no sleep problems at any wave. Fixed effects ordinary least squares (OLS) models tested in our sensitivity analyses suggest that this exclusion may lead Poisson models to overestimate associations between selected independent variables and the severity of sleep problems, though the overall patterns in these OLS models aligned with those identified through Poisson models. Additionally, as mentioned previously, we used the mathematical procedures recommended in Vaisey and Miles [39] to test key assumptions of fixed effects modeling, namely the assumption of consistent time trajectories and the assumption of unidirectional causation with no selection into treatment. While neither assumption was violated to a statistically significant degree, tests for reciprocal causation are sensitive to misspecification in the lag period of causal effects, so reciprocal or reverse effects occurring over a shorter span than 1 year are not captured by our analyses. It should be noted that threats to causal inference do linger-in particular, unobserved variables that change over time within individuals might confound the relationships under analysis or reciprocal causal relationships may play out over periods that do not coincide with the lag structure of the UCNets data.

\section{Conclusions}

In summary, the findings provide causal evidence that network support affects sleep quality, both directly and via moderation of stressful life events, enabled by the three waves of panel data available in the UCNets data set. Despite the limitations discussed above, this approach represents a significant advance over the causal contributions of standard regression analyses.

Focusing on network sufficiency was another key contribution: networks have both direct and indirect effects on sleep quality, and each network characteristic has its own pattern. The particular network tie-romantic relationships - were also predictive of trouble sleeping through their ability to add protection to sleep quality in the presence of stressful events. This study underscores important, interdependent relationships between personal networks, negative life events, and sleep quality. Lastly, the dual cohort structure of the UCNets study and the ability to distinguish between positions along the life course is rare in sleep studies. While the desire for more practical support was predictive of poor sleep quality for both young and older adults, indicative of underlying stress, we find that young adults experienced a buffering effect of having supportive network ties.

Our results have important clinical implications and suggest that efforts to improve sleep quality should address personal networks and the support they provide, especially during periods of stress, and particularly among young adults. The literature on treatment for chronic insomnia points to a combination of pharmacological, physical, and behavioral assessments and cognitive approaches [17]. To this, we add consideration of the social context of the person's life, particularly negative life experiences and changes in personal relationships, as well as therapies that assist patients with chronic sleeping difficulties in developing a network that meets their social support needs.

\section{Acknowledgments}

The authors wish to thank the anonymous reviewers for their feedback on an earlier version of this manuscript.

Funding: This work was supported by funding from the National Institutes of Health (AG059950, PI: S.C.; AG041955, PI: Fischer).

\section{Compliance with Ethical Standards}

Authors' Statement of Conflict of Interest and Adherence to Ethical Standards The authors declare that they have no conflicts of interest.

Authors' Contributions All authors helped conceive of the research ideas and hypotheses. S.C. lead paper writing and constructed the 
figures. E.R. lead the statistical analyses and assisted in writing. M.Z. lead the literature review and assisted with paper writing and editing. L.L. assisted in paper writing and editing and oversaw data collection efforts.

Ethical Approval All study procedures received prior approval from the Office for Protection of Human Subjects from the University of California, Berkeley.

Informed Consent All participants gave written or verbal informed consent prior to any study procedures.

\section{References}

1. Small ML. Someone To Talk To. New York, NY: Oxford University Press; 2017.

2. Chung J. Social support, social strain, sleep quality, and actigraphic sleep characteristics: Evidence from a national survey of US adults. Sleep Health. 2017;3:22-27.

3. Kent RG, Uchino BN, Cribbet MR, Bowen K, Smith TW. Social relationships and sleep quality. Ann Behav Med. 2015;49:912-917.

4. Liu X, Liu C, Tian X, et al. Associations of perceived stress, resilience and social support with sleep disturbance among community-dwelling adults. Stress Health. 2016;32:578-586.

5. Portela LF, Kröning Luna C, Rotenberg L, et al. Job strain and self-reported insomnia symptoms among nurses: What about the influence of emotional demands and social support? Biomed Res Int. 2015;2015:820610.

6. Nicholas CL, Jordan AS, Trinder J. The functions of sleep. In: Antic N, Rajaratnam S, Naughton M, Mansfield DR, eds. Sleep Medicine. Australia: IP Communications; 2017.

7. Tempesta D, Socci V, De Gennaro L, Ferrara M. Sleep and emotional processing. Sleep Med Rev. 2018;40:183-195.

8. Liu Y, Wheaton AG, Chapman DP, Cunningham TJ, Lu H, Croft JB. Prevalence of healthy sleep duration among adultsUnited States, 2014. MMWR Morb Mortal Wkly Rep. 2016;65:137-141.

9. Hawkley LC, Thisted RA, Masi CM, Cacioppo JT. Loneliness predicts increased blood pressure: 5-year cross-lagged analyses in middle-aged and older adults. Psychol Aging. 2010;25:132-141.

10. Gangwisch JE, Heymsfield SB, Boden-Albala B, et al. Sleep duration as a risk factor for diabetes incidence in a large U.S. sample. Sleep. 2007;30:1667-1673.

11. Irwin MR, Olmstead R, Carroll JE. Sleep disturbance, sleep duration, and inflammation: A systematic review and metaanalysis of cohort studies and experimental sleep deprivation. Biol Psychiatry. 2016;80:40-52.

12. McSorley VE, Bin YS, Lauderdale DS. Associations of sleep characteristics with cognitive function and decline among older adults. Am J Epidemiol. 2019;188:1066-1075.

13. Noble W, Spires-Jones TL. Sleep well to slow Alzheimer's progression? Science. 2019;363:813-814.

14. Grandner MA, Martin JL, Patel NP, et al. Age and sleep disturbances among American men and women: Data from the U.S. Behavioral Risk Factor Surveillance System. Sleep. 2012;35:395-406.

15. Yong LC, Li J, Calvert GM. Sleep-related problems in the US working population: Prevalence and association with shiftwork status. Occup Environ Med. 2017;74:93-104.

16. Roth T, Roehrs T. Insomnia: Epidemiology, characteristics, and consequences. Clin Cornerstone. 2003;5:5-15.
17. Krystal AD, Prather AA, Ashbrook LH. The assessment and management of insomnia: An update. World Psychiatry. 2019;18:337-352.

18. Cobb S. Presidential address - 1976. Social support as a moderator of life stress. Psychosom Med. 1976;38:300-314.

19. Waite LJ, Gallagher M. The Case for Marriage: Why Married People are Happier, Healthier, and Better Off Financially. New York, NY: Broadway Books; 2001.

20. Troxel WM, Buysse DJ, Matthews KA, et al. Marital/cohabitation status and history in relation to sleep in midlife women. Sleep. 2010;33:973-981.

21. Bookwala J. Confidant availability (In)stability and emotional well-being in older men and women. Gerontologist. 2017;57:1041-1050.

22. Dean A, Lin N. The stress-buffering role of social support. Problems and prospects for systematic investigation. J Nerv Ment Dis. 1977;165:403-417.

23. Guastella AJ, Moulds ML. The impact of rumination on sleep quality following a stressful life event. Pers Individ Dif. 2007;42:1151-1162.

24. Morin CM, Rodrigue S, Ivers H. Role of stress, arousal, and coping skills in primary insomnia. Psychosom Med. 2003;65:259-267.

25. Healey ES, Kales A, Monroe LJ, Bixler EO, Chamberlin K, Soldatos CR. Onset of insomnia: Role of life-stress events. Psychosom Med. 1981;43:439-451.

26. Matsumoto $\mathrm{S}$, Yamaoka $\mathrm{K}$, Inoue $\mathrm{M}$, Inoue $\mathrm{M}$, Muto $\mathrm{S}$; Teikyo Ishinomaki Research Group. Implications for social support on prolonged sleep difficulties among a disasteraffected population: Second report from a cross-sectional survey in Ishinomaki, Japan. PLoS One. 2015;10:e130615.

27. Cacioppo JT, Hawkley LC, Berntson GG, et al. Do lonely days invade the nights? Potential social modulation of sleep efficiency. Psychol Sci. 2002;13:384-387.

28. Cohen S, Wills TA. Stress, social support, and the buffering hypothesis. Psychol Bull. 1985;98:310-357.

29. Cohen S, Mermelstein R, Kamarck T, Hoberman HM. Measuring the functional components of social support. In: Sarason IG, Sarason BR, eds. Social Support: Theory, Research and Applications. Dordrecht, Netherlands: Springer Netherlands; 1985:73-94.

30. Zimet GD, Dahlem NW, Zimet SG, Farley GK. The multidimensional scale of perceived social support. J Pers Assess. 1988;52:30-41.

31. Pressman SD, Matthews KA, Cohen S, et al. Association of enjoyable leisure activities with psychological and physical well-being. Psychosom Med. 2009;71:725-732.

32. Hall ED, Meng J, Reynolds RM. Confidant network and interpersonal communication associations with depression in older adulthood. Health Commun. 2020;35:872-881.

33. Child ST, Lawton L. Loneliness and social isolation among young and late middle-age adults: Associations with personal networks and social participation. Aging Ment Health. 2019;23:196-204.

34. Tempesta D, Curcio G, De Gennaro L, Ferrara M. Longterm impact of earthquakes on sleep quality. PLoS One. 2013;8:e55936.

35. Zitting KM, Münch MY, Cain SW, et al. Young adults are more vulnerable to chronic sleep deficiency and recurrent circadian disruption than older adults. Sci Rep. 2018;8:11052.

36. Pow J, King DB, Stephenson E, DeLongis A. Does social support buffer the effects of occupational stress on sleep quality among paramedics? A daily diary study. J Occup Health Psychol. 2017;22:71-85.

37. Fischer CS, Bayham L. Mode and interviewer effects in egocentric network research. Field Methods. 2019;31:195-213. 
38. Halaby CN. Panel models in sociological research: Theory into practice. Annu Rev Sociol. 2004;30:507-544.

39. Vaisey S, Miles A. What you can - and can't - do with threewave panel data. Sociol Methods Res. 2017;46:44-67.

40. Allison PD, Waterman RP. Fixed-effects negative binomial regression models. Sociol Methodol. 2002;32:247-265.

41. Allison PD. Fixed Effects Regression Models. SAGE Publications; 2009.

42. Jacobson NC, Lord KA, Newman MG. Perceived emotional social support in bereaved spouses mediates the relationship between anxiety and depression. J Affect Disord. 2017;211:83-91.

43. de la Vega R, Molton IR, Miró J, Smith AE, Jensen MP. Changes in perceived social support predict changes in depressive symptoms in adults with physical disability. Disabil Health J. 2019;12:214-219.

44. Chen JH, Lauderdale DS, Waite LJ. Social participation and older adults' sleep. Soc Sci Med. 2016;149:164-173.
45. Chapman DP, Wheaton AG, Perry GS, Sturgis SL, Strine TW, Croft JB. Household demographics and perceived insufficient sleep among US adults. $J$ Community Health. 2012;37:344-349.

46. Chen JH, Waite LJ, Lauderdale DS. Marriage, relationship quality, and sleep among U.S. older adults. J Health Soc Behav. 2015;56:356-377.

47. Lee JH, Chopik WJ, Schiamberg LB. Longitudinal associations between marital quality and sleep quality in older adulthood. J Behav Med. 2017;40:821-831.

48. Lee S, Martire LM, Damaske SA, et al. Covariation in couples' nightly sleep and gender differences. Sleep Health. 2018;4:201-208.

49. Lawton L, Wilson W. Comparing facebook and addressbased sampling to recruit young adults. Paper presented at: Annual Meeting of the Pacific Association for Public Opinion Research; May 16-19, 2018; Denver, CO. 